\title{
Producción científica brasileña en España: documentación de las tesis doctorales*
}

Maria de Jesus Nascimento

Professora da Universidade do Estado de Santa Catarina (Udesc). Mestre em Ciência da Informação pelo IBICTUFRJ/ECA. Doutora em ciencia da Informação Universidad Complutense de Madrid, España.

E-mail:mjn@central148.com.br

\section{Resumen \\ Estudio bibliométrico aplicado a las tesis doctoraels de brasileros, presentadas en universidades españolas en el período de 1992 a 1995, cuyo análisis estadístico se efectúa en tres etapas: identificación de las tesis; caracterización de los doctores; y análisis de citación de los dos grupos de tesis más productivos, del área de ciencias humanas de la Universidad Complutense y del área de ciencias y tecnología de la Universidad Politécnica de Madrid. Además de investigar y registras las tesis, se concluye que la presencia de los brasileros en universidades españolas ha contribuido, si bien que modestamente, para el conocimiento científico en general, para la producción científica española $y$, principalmente, para una mayor cooperación entre los investigadores brasileros $y$ españoles.}

\section{Palabras clave}

Producción científica; Tesis; Análisis de citación.

\section{Brazilian scientific producction in Spain: quantitative analysis applied to doctorate theses}

\begin{abstract}
Bibliometric study applied to Brazilian doctorate theses at Spanish Universities from 1992 to 1995, whose statistic analysis was put into effect in three stages: theses identification; doctorate student features; and citation analysis of the two more productive groups in the Human Science area at Complutense University and in the Science and Technology area at Polytechnic University of Madrid. Besides the theses survey and registration it was noticed that the presence of Brazilians at Spanish Universities, even though moderately, contributed for scientific knowledge in general, for Spanish scientific production and mainly, for more cooperation between Brazilian and Spanish researchers.
\end{abstract}

\section{Keywords}

Scientific production; Thesis; Citation analysis.

Artigo aceito para publicação em 24/08/99

\section{INTRODUCCIÓN}

"¿Cómo encontrar la sabiduría que hemos perdido en el conocimiento? ¿Cómo encontrar el conocimiento que hemos perdido en la información?" Georg Eliot ${ }^{* *}$

Preguntas de este tipo pueden ser fácilmente contestadas: en la literatura, es decir, en la bibliografía del área específica del conocimiento, ya que el saber está directamente relacionado a la investigación y a la transmisión del conocimiento a través de la información documentada.

Partiendo del supuesto de que la tesis doctoral es un trabajo académico de iniciación científica, marco inicial de la especialidad de un investigador, constituyendose una fuente de información que refleja el logro en su propio campo del saber, se considera de gran importancia la recopilación y su análisis, para el estudio de una determinada producción científica.

Las características metodológicas de las tesis doctorales, la orientación del director y la evaluación del tribunal garantizan el nivel de calidad de este tipo de trabajo de investigación que constituye un bueno indicador, no sólo cuantitativo sino cualitativamente de la producción científica.

Muchos brasileños dejan el país en busca de nuevos conocimientos en universidades extranjeras. En la mayoría son profesionales - profesores e investigadores de órganos gubernamentales o universidades del gobierno y lo hacen con becas de instituciones brasileñas, así como de órganos internacionales para cursar el doctorado en universidades del Primer Mundo.

Estos profesionales desarrollan sus estudios y trabajos de investigación en los países anfitriones, dejando en ellos gran parte de su colaboración en el terreno del conocimiento humano, que pasa a formar parte de la

\footnotetext{
* Artigo elaborado com base nos resultados da tese de doutorado "Producción Científica Brasileña en España: estudio bibliométrico aplicado as las tesis doctorales" defendida na Unversidad Complutense de Madrid.

** Georg Eliot. En: AMAT, Nuria. De la información al saber. Madrid: Fundesco, 1990, p. 47.
} 
producción científica del país donde está cursando el doctorado, sin que estos conocimientos sean identificados como una colaboración de un brasileño.

Dando por cierto que un gran número de brasileños van a universidades españolas para cursar el doctorado, y que la tesis es indispensable para la conclusión de dichos cursos, se eligió la tesis como instrumento de estudio para valoración de la producción científica brasileña en España.

La falta de control y organización de las tesis, o mejor la inexistencia de bases de datos de tesis brasileñas en España, es un hecho revelador. Por otro lado, los estudios bibliométricos, en casi en su totalidad, están más preocupados por los artículos de revistas, y poco o casi nada se ha hecho utilizando las tesis.

Por eso el presente estudio trata de documentar* la producción científica brasileña en España a través de las tesis doctorales de brasileños leídas en universidades españolas, en el periódo comprendido entre 1980 y 1994.

Con el objetivo de conocer mejor esa producción, se intenta caracterizar el perfil del investigador brasileño en España, es decir, se constata quiénes son los doctores, que tesis elaboraron, dónde y cuando han desarrollado sus investigaciones, y cuáles las características de la literatura que han usado como base de su trabajo, o sea, las características de las citas de las tesis.

\section{PRODUCCIÓN CIENTÍFICA BRASILEÑA EN EUROPA}

La ciencia y la tecnología ejercen un gran papel en la historia del presente siglo, por su influencia directa en el desarrollo económico, político, cultural, tanto en los países del Primer Mundo, como en los clasificados como tercer mundo, que si no producen ciencia, sufren las consecuencias y son usufructuarios de las ventajas proporcionadas por ellas.

El último tercio de este siglo está marcado por el fenómeno económico de la internacionalización de los mercados y economías de los países más avanzados, como el caso de la Comunidad Europea (CE) y el más reciente Plan del Mercosur en América Latina.

\footnotetext{
* Documentar en el sentido de «informar con la ayuda de documento» y considerando que un documento sólo existe cuando se utiliza como tal. SAGREDO FERNÁNDEZ, Félix y IZQUIERDO, José Ma․ La concepción ordinaria de Ciencia de la Documentación. En: LÓPEZ YEPES, J. (compilador) Fundamentos de Información y Documentación. Madrid: EUDEMA, 1989, p. 56.
}

A la vez de la globalización y por el carácter de internacionalidad de la ciencia es muy difícil determinar el "origen nacional" de un saber científico. Los científicos publican siempre en países que no son los suyos como ha demostrado Silgleton, ${ }^{*}$ con el fenómeno de la "Transpatriation".

Los países en vías de desarrollo también son productores de información científica, pero, muchas veces, su propia comunidad académica no lo valora; por el contrario, subutiliza la propia producción nacional.

En el caso de Brasil, este fenómeno ocurre frecuentemente desde hace muchos años. Muchos son los estudios hechos en diferentes áreas y épocas cuyos resultados coinciden en que hay una gran evasión de la producción científica brasileña.

Los resultados del estudio de Nascimento, ${ }^{* *}$ sobre la Producción científica brasileña en el área nuclear constatan el fenómeno de la "Transpatriation" del conocimiento, pero sobre todo se puede deducir que los investigadores brasileños están en desventaja en relación a sus colegas extranjeros, no sólo por el status de productores de información, sino por las posibilidades de acceso a la información de su propio país que muchas veces, están publicadas en revistas extranjeras que no llegan a todas las instituciones brasileñas.

La literatura científica brasileña, según la clasificación de Meneghini*** $^{* *}$ es de dos tipos: endógena y exógena. Los trabajos endógenos son los realizados integral o parcialmente (en este caso con la colaboración de investigadores extranjeros) en Brasil. Los exógenos corresponden a los artículos producidos por investigadores brasileños en instituciones extranjeras, la gran mayoría en Estados Unidos o Europa.

El intercambio de experiencias e informaciones entre países cuyo nivel de desarrollo no es equivalente constituye una contradicción, ya que no siempre las experiencias que surtieron efecto en países desarrollados son adecuadas a la realidad de los países menos desarrollados.

\footnotetext{
" SINGLETON, Alen. International publication: change patterns. Pysics Bulletin. aug. 1975. p. 354-5.

** NASCIMENTO, Maria de Jesus. Estudo da produção científica brasileira na área nuclear no período 1970/1979. Rio de Janeiro: IBICT. 1983, 209p. (Dissertação de Mestrado)

*** MENEGHINI R. y FONSECA L. «Índice alternativo de avaliação da produção científica em bioquímica no Brasil». Ciência e Cultura. v. 42, n.9, p. 632.1990
} 
En la mayoría de los países latinoamericanos, la infraestructura universitaria local no corresponde totalmente a las necesidades internas. De ahí, la necesidad de buscar estos medios en universidades del extranjero, principalmente en lo que se refiere a la formación académica de los investigadores. Esta es la principal razón que lleva a profesores e investigadores a buscar los cursos de doctorado en universidades del Primer Mundo.

La producción científica y tecnológica de un país está determinada por recursos económicos que el gobierno destina a las actividades de Investigación y Desarrollo $(\mathrm{I}+\mathrm{D})$ y por la inversión en capital humano investigador. Y uno de los factores que impulsaron el progreso de la ciencia y de la técnica en la actualidad fue y es precisamente el intercambio de informaciones. Para una nación desarrollarse científica y tecnológicamente debe dar prioridad a la formación de investigadores, mejorar el sistema universitario y facilitar el flujo e intercambio de información científica y tecnológica.

En un estudio de la producción de información científica y tecnológica de los países latinoamericanos en la base de datos del Instituto de Información y Documentación en Ciencia y Tecnología (ICYT), del Consejo Superior de Investigaciones Científicas (CSIC), de España, sólo diecinueve países están representados en con sólo el 2,6\% del total de dicha base.

En la aportación científica de estos países, Brasil es el sexto país de mayor producción en las revistas españolas, después de Argentina, Chile, Cuba, Venezuela y Méjico." Pero según Sancho, ${ }^{* *}$ Brasil ocupa el primer lugar, es el país más productivo en América Latina y el que proporcionalmente ha realizado mayor número de trabajos en colaboración, publica con casi todos los países principalmente Argentina, Chile y México.

En relación a la publicación de artículos de revista, Brasil está en primer lugar en el rango de los países latinoamericanos que publican en Europa. De los 8.044 artículos latinoamericanos publicados en Europa, Brasil fue el mayor productor con el 10,5\%, pero en relación a

\footnotetext{
* MARTÍN SEMPERE, M. J.; URDIN CAMINOS, M. C. «Producción científica de los países Latino Americanos a través de las revistas españolas de ciencia y tecnología durante el período de 1983-1988. Rev. Esp. Doc. Cient. v. 14, n. 2, 1991, pp. 143-153.

** SANCHO, R. et. al. A study of scientific co-operation between South, Central America and Caribian Countries. Madrid: Centro de Información y Documentación Científica (CSIC). p. 166-76.
}

España, de los 2.152 artículos latinoamericanos, sólo el $3,5 \%$ son publicaciones de brasileñas.

Por otro lado, los datos que presenta DIEZ HOYO, ${ }^{* *}$ cuanto a publicación de libros latinoamericanos en España, Brasil publica sólo el 1,6\% del total de 2.225 libros, el índice más bajo en relación a los otros países donde Brasil ha publicado, como por ejemplo, en Francia, Brasil está en tercer lugar con el 13,4\%; también ocupa el tercer lugar en El Reino Unido y el segundo en Alemania.

El bajo porcentaje de libros que publica Brasil en España en relación a los demás países latinoamericanos puede ser justificado, en parte, por el factor idioma, ya que los demás países latinoamericanos son de habla española, mientras Brasil es de lengua portuguesa.

En el análisis del "Repertorio de Tesis Europeas sobre América Latina", del REDIAL, ${ }^{* * *}$ que reseña las tesis sobre América Latina, leídas en Europa, en el período de 1980 a 1990, hemos constatado - en el índice geográfico - que Brasil es el país con el mayor número de tesis, con un total de 555 . De éstas el 71\% fueron leídas en universidades de Francia, el 12\% en Inglaterra, el 11,6\% en Alemania, el 2,5\% en Austria y sólo el 2,3\% en España seguida del 0,5\% en Bruselas.

Estos datos deben ser observados con mucha precaución teniendo en cuenta que el REDIAL registra como tesis brasileñas sólo las que llevan la palabra «Brasil» en su título. Por lo tanto, éste no es un indicador de los más significativos.

Considerando la política de postrado brasileña de los últimos años, que ha aumentado y mejorado los cursos de doctorado en Brasil, dónde se leyeron siete mil tesis de doctorado y de «Mestrado» por año, en el periódo comprendido entre 1990 y $1992^{* * * *}$ al mismo tiempo que mantiene las becas para el extranjero, es de esperar que el número de tesis escritas por brasileños en España si no crece por lo menos se mantenga estable.

\footnotetext{
* DIEZ HOYO, M.Del C.»Balance de la producción científica europea de monografías y artículos de revistas sobre América Latina entre 1980 y 1989. REDIAL, n. 2, 1993, pp. 7-20.

** Ibíd. Ídem

*** REDIAL. Red Europea de Información y Documentación sobre América Latina. Repertorio de tesis Europeas sobre América Latina 1980. 1990. Madrid: Quinto Centenario, 1992, p. 407.

**** “CNPq regista 23 mil cientistas”. Jornal da Ciência hoje. SBPC, ano 9, n. 303, p. 5, Rio de Janeiro, 1994.
} 
LAS TESIS DE DOCTORADO: SU CONTROL Y ACCESO

Es un hecho universalmente aceptado que la investigación científica depende de la calidad de las Universidades y de las Instituciones Profesionales y de Investigación de cada país. Las Instituciones educativas de alto nivel han aceptado el papel de preparar a los futuros científicos y conceder el grado de Doctor a aquellos que demuestren estar capacitados para llevar a cabo investigaciones de alta calidad por sus propios medios.

La tesis doctoral, o de $\mathrm{PhD}$ (Doctor of Phylisophy), se hace con vistas a la actividad académica, es así, considerada como el primer trabajo, que inicia la vida académica de un investigador. Es un trabajo original de investigación, que sirve para comprobar que el doctorando es un estudioso capaz de hacer avanzar el conocimiento en su área de actuación. Orientada por un Director, juzgada y debidamente aprobada por un tribunal competente, confiere al investigador el título de Doctor.

La tesis es un trabajo de creación, inédito, una investigación rigurosa que se hace después de la realización del tercer ciclo y por lo tanto es el prólogo de la especialización posterior de un investigador.*

La tesis es un tipo de documento primario, que forma parte de la "literatura gris" y constituye una rica fuente de información aunque su divulgación es limitada y el número de ejemplares, además de los exigidos por la universidad, dependen mucho más del doctorando que de cualquier otro factor. Por sus características de trabajo académico, de carácter único, no periódico y en la mayoría de los casos no editada, es de difícil difusión y acceso muy restringido. El acceso a las tesis inéditas se limita, muchas veces, a biblioteca de la escuela, y están sujetas a las reglas de cada facultad. En algunas facultades, tampoco se facilita el acceso a este rico material bibliográfico que se confunde en el medio del gran número de publicaciones españolas. Hay incluso bibliotecas en España que no permiten la consulta de las mismas.

La importancia de las tesis doctorales en el mundo académico, así como su colaboración para el desarrollo de una determinada producción científica es indiscutible, pero poco o casi nada se hace, o se investiga sobre este tipo de documento. Todavía no existe en las universidades españolas una preocupación en ordenar las tesis especificando el país de origen del autor o el tema que tratan.

\footnotetext{
* FUENTES i PUJOL, Mํㅡㄹ Eulàlia. Documentación científica e información. Metodología del trabajo intelectual y científico. Barcelona: Promociones y Publicaciones universitarias. 1992, p. 21.
}

En lo que se refiere a las tesis Doctorales, según Albuquerque* en España, hasta 1992 no parece existir aún ninguna institución que sea depositaria de las tesis doctorales sobre América Latina.

La tentativa de una mayor y mejor cooperación científica entre la comunidad europea y la comunidad latinoamericana, llevó a la fundación en 1989 de la Red Europea de Información y Documentación sobre América Latina (Redial).**

España como participante de esta red ha desarrollado un plan de cooperación bibliotecaria de ámbito internacional, ya que quiere presentarse como el nexo privilegiado entre Europa y América Latina. Por eso algunas tentativas han sido hechas y en 1992, año que coincidió con las conmemoraciones del Quinto Centenario del Descubrimiento de América y con la concretización de la Comunidad Económica Europea (CCE), de la cuál España es parte, se publicó el "Repertorio de Tesis Europeas sobre América Latina 1980-1990”, con el objetivo de mejorar la accesibilidad de los recursos bibliográficos sobre América Latina. ${ }^{* * *}$

Para que España actúe como nexo de unión entre el ámbito latinoamericano y el europeo, en el campo de la información Iberoamericana, varias iniciativas están siendo llevadas a cabo principalmente en lo que se refiere al registro, control y divulgación de la literatura latinoamericana. Se está poniendo de relieve dos hechos que marcan el interés en recuperar la memoria de la información de latinoamericana:

- Se ha creado un catálogo colectivo de fondo antiguo (siglos XVI al XIX).

- Se han creado tres bases de datos, editadas en CD-ROM, con la denominación "Bibliotecas sin fronteras", que son: base de datos de monografías, de artículos y de tesis doctorales.

Las tesis sobre América Latina, en europa, empieza a disminuir considerablemente en los últimos años, llegando a un 50\% de reducción en Francia, según una encuesta llevada a cabo por Duport ${ }^{* * * *}$ en siete países

\footnotetext{
* AlBUQUERQUE, Francisco. Prólogo. En: Repertorio de tesis europeas sobre América Latina 1980-1990 (REDIAL) Madrid: Quinto Centenario. 1992.

${ }^{* *}$ REDIAL. Op. cit.

${ }^{* * *}$ REDIAL Op. cit.

**** DUPORT, C. «Tesis Doctorales europeas sobre América Latina, 1980-1989». Datos bibliométricos y algunos comentarios.» REDIAL, 1992. p. 21.
} 
miembros de Redial (Austria, Bélgica, Francia, Países Bajos, Reino Unido y Suecia). Pero, éstas han aumentado progresivamente en España, tal vez como resultado del interés de los españoles en aumentar los lazos con América Latina.

En España poco o casi nada se conoce de las tesis de brasileños leídas en este país. También en Brasil, el control y difusión de las tesis todavía no está institucionalizado, poco se conoce de las tesis leídas en el extranjero. El "Instituto Brasileiro de Informação em Ciencia e Tecnoliga" (IBICT) ha creado un "Banco de Dissertação e Teses", pero este documenta más las tesis producidas dentro de las fronteras brasileñas.

Así al analizar las tesis se pretende documentar y proporcionar elementos de reflexión sobre parte de la producción científica brasileña en España.

\section{IDENTIFICACIÓN DE LAS TESIS Y RECOPILACIÓN DE LOS DATOS}

Se ha llevado a cabo este estudio utilizándose técnicas cuantitativas, para el análisis de las tesis doctorales, así como de los doctores, autores de dichas tesis.

Inicialmente se han recopilado las tesis brasileñas aceptas en las universidades españolas, en todas las áreas del conocimiento y se han hecho el análisis de las características de las tesis y de los doctores, así como de las citas de las tesis del área de Ciencias Humanas de Universidad Complutense y de Ciencias y Técnicas de la Universidad Politécnica de Madrid.

El período de tiempo analizado corresponde a quince años y abarca desde 1980 hasta 1994 . Se ha analizado un período de tiempo, lo más largo posible, para tener una muestra más representativa.

Se ha elegido analizar las tesis a partir del año 1980 por varias razones. En primer lugar el II PND - Plano Nacional de Desenvolvimento - durante el periódo de 1975/79 tenía como objetivo principal la institucionalización de los cursos de postgrado en Brasil, consecuentemente la necesidad de un mayor número de doctores hacia actuar en dichos cursos, así estimuló la salida de muchos brasileños para los cursos de doctorado en el extranjero y, obviamente, el resultado de eso hecho se obtiene sólo en la década de los 80. También el año de 1980 coincide con el primer año de registro de las tesis sobre América Latina publicadas por el Redial. Además, no se ha encontrado registros de tesis, antes de esta fecha, en número suficiente para realizar un análisis cuantitativo.
Por no existir aún ninguna institución en España que sea depositaria de las tesis doctorales brasileñas y tampoco los registros de las universidades no haceren distinción de la nacionalidad de los autores de las tesis doctorales, ha sido muy difícil la identificación de dichas tesis. Para eso, fue necesario el examen de las más variadas fuentes y fondos.

La identificación de las tesis brasileñas en España se ha realizado mediante la consulta a publicaciones de resúmenes de Tesis, catálogos de bibliotecas y archivos, listados de base de datos, instituciones brasileñas e internacionales que conceden becas para cursos de doctorado, los sectores de doctorado de las universidades y a través de informaciones informales ya que ningún de estas fuentes por si solo documentan de forma completa las tesis de los brasileños en España.

Inicialmente se hizo la búsqueda en el Repertorio de Tesis Europeas sobre América Latina, ${ }^{*}$ en el Catálogo de Tesis Doctorales del Consejo de Universidades, ${ }^{* *}$ en la bases de datos de tesis doctorales de universidades españolas Teseo del Ministerio de Educación y Ciencia, ${ }^{* * *}$ y en la base de datos del Centro de Información y Documentación Científica (Cindoc) del Consejo Superior de Investigaciones Científicas (CSIC).

Se hizo también una consulta al catálogo de antiguos becarios, del Instituto de Cooperación Iberoamericana (ICI) ${ }^{* * * *}$ y al Servicio de Programación Educativa del Instituto Español de Emigración, que concede becas para estudios de postgrados a los hijos de emigrantes y ayuda a la publicación de tesis doctorales.

Se consultaron también los listados de becarios de las instituciones brasileñas que conceden becas para estudios de postgrado como: Capes; CNPq; Embrapa y Fapesp. ${ }^{* * * * *}$

\footnotetext{
* REDIAL. Op. cit.

** CONSEJO DE Universidades. Secretaria General. Tesis Doctorales. Madrid. 1976/1988 (3 tomos). Y 1989/1990.

${ }^{* * *}$ MINISTERIO DE EDUCACIÓN Y CIENCIA. Centro de Proceso de datos. Relación de tesis brasileñas 1980 - 1984. Madrid: MEC, 1994. 1995.

***** INSTITUTO DE COOPERACIÓN IBEROAMERICANO - ICI. Catálogos de antiguos becarios 1980-1991. Madrid: Agencia Española de Cooperación Internacional - Becas.

${ }^{* * * * *}$ La CAPES subsidia 22.000 becarios y el CNPq 42.000 para cursos de Mestrado y Doctorado, tanto en Brasil como en el extranjero. Datos del Jornal da Ciencia Hoje. Rio de Janeiro. Ano 9, no 307, set. 1994.

CAPES - Coordenação de Aperfeiçoamento de Pessoal de nível Superior.

CNPq - Conselho Nacional de Desenvolvimento Científico e Tecnológico. EMBRAPA - Empresa Brasileira de Pesquisa Agropecuária.

FAPESP - Fundação de Amparo a Pesquisa de São Paulo.
} 
También se procedió a la búsqueda de nombres de brasileños en la biblioteca del "Centro de Referencia sobre Brasil" y en los archivos de la secretaria de la "Casa do Brasil". Además de eso se consultaron las Actas de Reunión y Registro de socios de la Asociación de posgraduados brasileños en España (APBE), con sede en el Colegio Mayor de la «Casa do Brasil» en Madrid.*

En las universidades sitas en la Comunidad de Madrid: Universidad Complutense de Madrid (UCM), ${ }^{* *},{ }^{* * *}$ Universidad Politécnica de Madrid (UPM), ${ }^{* * * *}$ Universidad Autónoma de Madrid (UAM); Universidad de Enseñanza a Distancia (UNED); y Universidad de Alcalá de Henares además de consultar los Resúmenes de Tesis, se hizo la visita en cada Escuela o Facultad y se efectuó una búsqueda en los sectores de Doctorados, secretaría, archivos y ficheros de la biblioteca.

Tratándose de universidades de otras Comunidades se remitió por correo una solicitud de nombres de brasileños que habían leído sus tesis en dichas universidades, así como el título de las tesis, el nombre del director y la fecha de lectura.

La identificación de los datos que caracterizan al doctorando, cuando no fue posible identificarlos en la propia tesis, se intentó en las secretarías de las facultades o por medio de la comunicación informal.

\section{LIMITACIONES DEL ESTUDIO}

Sin pretensiones de llevar a cabo un estudio completo y perfecto, aún afrontando las limitaciones y dificultades, se ha intentado documentar al máximo número de tesis posible.
No se puede garantizar que todas las tesis de brasileños presentadas en universidades españolas estén registradas en este estudio, pero se ha conseguido registrar un considerable número de ellas, teniendo en cuenta que algunas universidades españolas no contestaron positivamente a la solicitud de nombres de brasileños ni dieron a conocer los respectivos títulos de las tesis aprobados en dichas universidades. Esta negativa se fundamenta en razones de protección a la personal o por no haber tesis brasileñas en dichas universidades.

Algunas universidades alegan "restringir al máximo este tipo de información, en cumplimiento en buena parte de la Ley Orgánica 5/1992”. Otras contestaron que los ficheros no recogen la nacionalidad de los doctorandos, o bien que no tienen autorización expresa de los alumnos para divulgar sus nombres.

En España, además de no tener ningún tipo de registro de tesis especificando la nacionalidad del autor, la protección a la persona y la normativa de algunas facultades en relación al sigilo de las tesis dificultaron y retrasaron el desarrollo de ésta investigación.

Este estudio está limitado por varias razones tanto en lo que se refiere a la muestra, como al sujeto y su análisis, pero estas limitaciones no invalidan su importancia como indicador de los patrones de la Producción Científica Brasileña en España.

La muestra es cuantitativamente representativa, aunque muy heterogénea, ya que incluye las carreras de dos áreas del conocimiento humano. Con excepción de la especialidad de Agrónomos que tiene un bueno número de tesis, las demás carreras no forman un corpus suficiente para que se puedan inferir conclusiones sobre la especialidad.

El estudio se limita al análisis cuantitativo de las características extrínsecas de las tesis, no se ha intentado ningún tipo de juicio de valor o de calidad del contenido.

Aunque se ha elegido como instrumento de análisis las tesis elaboradas por brasileños, no se pueden menospreciar las tesis sobre temas brasileños escritas por doctores que no se haya comprobado su nacionalidad. A efecto de este estudio se considera tesis brasileña, además de las elaboradas por aquellos que hayan nacido en Brasil, las elaboradas por quienes tenga pasaporte brasileño, hayan desarrollado sus estudios de grado en universidades brasileñas o trabajen en una institución brasileña.

\footnotetext{
* La «Casa do Brasil» es una institución cultural del gobierno brasileño, inaugurada en 1962 y vinculada al Ministerio de Relaciones Exteriores. La casa mantiene, desde su fundación, un Colegio Mayor Universitario adscrito a la Universidad Complutense de Madrid.

${ }^{* *}$ El número de tesis leídas en la UCM durante los años 60 y posteriores, pasan de 20.000, según los registros del Archivo de Tesis, que comienza la numeración del registro de las tesis «modernas» en este periódo.

*** UNIVERSIDAD Complutense de Madrid. Resumen de tesis doctorales. 1985, 1986, 1987, 1988, 1989, 1990, 1991, 1992.

**** UNIVERSIDAD Politécnica de Madrid. Resumen de tesis Doctorales. Curso 1980/81, 1981/82, 1982/83, 1983/84, 1984/85 y 1991/92.
} 


\section{DESCRIPCIÓN DE LA POBLACIÓN}

Se han identificado 157 tesis leídas por brasileños en varias universidades españolas con el objetivo de tener una visión general de la producción científica brasileña en España.

De estas se han escogido una muestra de 82 tesis consecuentemente 82 doctores, autores de dichas tesis -, siendo 40 del área de Ciencias Humanas de la Universidad Complutense y 42 del área de Ciencia y Tecnología de la Universidad Politécnica de Madrid, las dos universidades y áreas mas productivas.

Las tesis de la muestra produjeron un total de 13.759 citas o referencias bibliográficas, siendo 7.744 del área de Ciencias Humanas y 6.015 del área de Ciencias y Técnicas.

\section{PROCEDIMIENTO ESTADÍSTICO DEL ANÁLISIS DE LOS DATOS}

Los datos recopilados fueron analizados a través de cálculos de porcentajes y testes estadísticos del programa SAS del Servicio de Informática de la Universidad Complutense de Madrid, levándose en cuenta tres fases distintas:

A - Identificación de las tesis brasileñas en España.

B - Caracterización de los doctores brasileños en España.

C - Análisis de las tesis y respectivas citas.

En la primera fase se han calculado porcentajes de los datos del total de las tesis, por fecha - año de lectura -, áreas y tema, por los títulos.

Para lo dos grupos de tesis - de Ciencias Humanas y Ciencias Técnicas se hizo la caracterización de los doctores en términos de sexo, edad, tiempo que les llevó para leer la tesis; beca y dispersión geográfica de origen de los autores.

En la ultima fase se hicieron los análisis cuantitativos referentes a las tesis y sus respectivas citas o referencias bibliográficas. Se han analizado las siguientes variables: medias de citas por tesis, lengua de publicación, tipo de documentos citados; edad de las citas, citas de directores y autocitas.

\section{RESULTADOS Y CONCLUSIONES}

Los resultados y las conclusiones que se exponen se restringen a las tesis documentadas y a la muestra analizada. El número de tesis brasileñas en universidades españolas es muy incipiente en la década de los ochenta y a partir del último año de esta década empieza una fase de crecimiento irregular pero en proporciones muy superiores a los años anteriores.

Este impulso en el crecimiento del número de tesis brasileñas en España puede ser una consecuencia de la política científica implantada en la Nova República con el Plano Nacional de Desenvolvimento (PND) para 1986-89, que trató de fortalecer la capacitación de los investigadores brasileños.

La Universidad Complutense de Madrid, con un 33,12\% del total, se destaca como la universidad más productiva de tesis brasileñas en España, principalmente en el área de Ciencias Humanas (CH) (tabla 1). La Universidad Politécnica de Madrid es la segunda mayor productora con un 29,30\% de las tesis recopiladas y ocupa el primer lugar en cuanto a las tesis del área de Ciencias Técnicas (CT).

\section{TABLA 1}

Total de tesis brasileñas en universidade españolas

\begin{tabular}{lccccc}
\hline Área/ & $\begin{array}{c}\text { Complutense } \\
33,12 \%\end{array}$ & $\begin{array}{c}\text { Politécnica } \\
29,30 \%\end{array}$ & $\begin{array}{c}\text { Outras } \\
* * 37,58 \%\end{array}$ & Total & $\%$ \\
\hline $\mathrm{CH}$ & $40 *(1)$ & - & 28 & 69 & 43,95 \\
$\mathrm{CT}$ & - & $42 *(4)$ & 18 & 64 & 40,76 \\
$\mathrm{CV}$ & 11 & - & 13 & 24 & 15,29 \\
TOTAL & 52 & 46 & 59 & 157 & 100 \\
\hline
\end{tabular}

* Tesis no analizadas por falta de acceso a las mismas.

** Universidades no analizadas por no hacer parte de la muestra.

El resto de las tesis, un 37,58\%, está distribuido en trece universidades de todo el país, destacada la Universidad de Barcelona con 22 tesis, que además de las dos áreas inclu yen el área de Ciencias Médicas y de la Vida (CV).

En la distribución por área del conocimiento, las tesis de CV representan sólo un 15,29\% y quedan en tercer lugar, con porcentajes muy inferior a las dos otras áreas: CT con un $40,76 \%$ y $\mathrm{CH}$, el área más productiva, con un 43,95\% del total. 
De esto se puede concluir que hay una fuerte tendencia de los brasileños a realizar el doctorado en España en las áreas de $\mathrm{CH}$ seguido de $\mathrm{CT}$ y eligen principalmente los doctorados de universidades sitas en Madrid.

En cuanto a la fecha de lectura de las tesis la mayor incidencia en $\mathrm{CH}$ y CT ocurrió en los años mas productivos: 1992 y 1993. El que puede deducirse que estas dos áreas colaboraron para el incremento de la producción de tesis brasileñas en España en dicho periódo.

En lo que se refiere a la temática de las tesis, de acuerdo con los datos del registro total, se desprende que la muestra fue la que determinó el resultado de que en $\mathrm{CH}$ existe un mayor número de tesis que tratan de temas brasileños, con un porcentaje de 59,42\% de su área, mientras se ha comprobado que las tesis de CT tratan más temas especializados, con un $57,8 \%$ y CV con el $75 \%$ del total en cada área respectivamente. Este resultado es muy comprensible considerando las características temáticas de éstas áreas, pero nada impide que temas especializados puedan ser aplicados a la realidad brasileña.

Con excepción de la Universidad Complutense y de la Universidad Politécnica, estos resultados se limitan a los títulos de las tesis, por falta de acceso a toda la populación, lo cual no garantiza al cien por cien el resultado, considerando que los títulos pueden no especificar exactamente que tratan de un tema brasileño cuando así lo hacen

En cuanto a la caracterización de los doctores, autores de las tesis de la muestra, es decir, de las tesis de $\mathrm{CH}$ de la Universidad Complutense y de las tesis del área de CT de la Universidad Politécnica, en general, el perfil que se puede diseñar es el de doctores de sexo masculino con edad de 35 años, profesores universitarios, con beca del CNPq o Capes que viene más del nordeste y sur de Brasil, empleando cuatro años para concluir el doctorado.

Pero se han encontrado diferencias significativas entre las dos áreas, así el perfil de los doctores brasileños difiere en cada una de las áreas. La diferencia de sexo no es tan grande en el área de $\mathrm{CH}$, con un 55\% del sexo masculino y un $45 \%$ del sexo femenino, como lo es en CT, con un porcentaje de $88,10 \%$ del sexo masculino y solo un $11,90 \%$ del sexo femenino. En resumen, hay un predominio del 71.95\% del sexo masculino en el total de doctores brasileños en las dos áreas.
La media de edad de los doctores brasileños en $\mathrm{CH}$ es de 38,47 años, siendo la edad mínima encontrada 29 años y la máxima de 60 años. Mientras que en Ciencias Técnicas la edad media de los doctores es de 38,33 años, siendo que la edad mínima encontrada 26 años y la máxima, 49 años. La edad mediana es de 35 años para los dos grupos.

La media del tiempo de duración del doctorado para el grupo de brasileños en $\mathrm{CH}$ es de 4,34 años, siendo el periódo mínimo encontrado de tres años y el máximo de siete, mientras en CT la media es de 3,83 años, con un período mínimo de dos años y un máximo de seis. La duración mediana es de cuatro años para los dos grupos.

La edad de los doctores del área de $\mathrm{CH}$ es más elevada, y emplean más tiempo en concluir el doctorado que los doctores de CT. El que se puede inferir que el tiempo de duración del doctorado depende del área y de la edad del doctorando.

En $\mathrm{CH}$ hay un predominio mayor de becarios del CNPq, con un 40\%, seguido de Capes, con un 20\%. Las otras instituciones (ICI, CSIC e Fapesp) concedieron sólo el $12,5 \%$ de las becas. El 27,5\% de los doctores restantes no se ha identificado si han obtenido o no una beca. En esta área hay un mayor número de doctores que no obtuvieron becas, y también un mayor número de los que obtuvieron dos becas.

En CT el número de becas de Capes Y CNPq es igual, cada institución con un 30\%, las otras instituciones (Embrapa, ICI y Fapesp) concedieron sólo un 16,67\%. El 23,33\% de los doctores restante no se ha identificado si ha obtenido o no una beca. En esta área es menor el número de doctores con dos becas y también es menor el número de doctores sin beca. Así se pude concluir que en CT la distribución de becas es más regular que en $\mathrm{CH}$, donde hay más disparidades.

Las dobles becas fueron siempre del ICI con una de las instituciones brasileñas. Considerando que en Brasil no está permitido disponer de dos becas para cursar el doctorado, queda investigar si estas becas han sido usufructadas al mismo tiempo o en períodos distintos.

En relación a la dispersión geográfica del origen de los doctores brasileños, la gran mayoría proviene del Nordeste brasileño, así se indica en el área de CT con un predominio de un 40,48\%, siendo que casi la mitad de ellos son de Paraíba, y sólo un 11,9 son del sur. Mientras en el cómputo del área de $\mathrm{CH}$ se ha constatado que hay mas doctores del sur con un $25 \%$, principalmente de Rio Grande do Sul. 
El Nordeste y Centro-Norte representan, cada una de estas regiones, un $15 \%$ de los doctores en $\mathrm{CH}$, mientras en CT las regiones Norte y Centro-Norte son poco representativas con bajos porcentajes, sólo un 2,38\% en cada una de ellas. De la región Sureste proviene 16,67\% de los doctores de CT y un $12,55 \%$ de $\mathrm{CH}$.

Además de muchas tesis sin identificación de la origen del autor, un 32,5\% en $\mathrm{CH}$ y un 16,19\% en CT, se han encontrado diferencias significativas en cuanto la distribución geográfica de los doctores en las dos áreas. Las discrepancias por regiones reflejan las diferencia que se ha constatado en las comunidades de cada región. Por eso no se puede inferir que ésta distribución refleja la preferencia por determinadas líneas de investigación, sino que esto ocurra, quizás como consecuencia de comunicaciones informales entre pares que ayudan en los trámites de los papeles de sus colegas que desean ingresar en la universidad española dónde éste ha hecho sus estudios de doctorado.

En cuanto al análisis de las 13.759 citas, las tesis del área de CH tienden más a hacer uso de éstas, con media de 193,6 que las tesis de CT con media de 143,2 citas por tesis.

Las tesis de las dos áreas analizadas difieren en cuanto al uso de la lengua, mientras que en $\mathrm{CH}$ hay una mayor predominio de citas en español con un $50,87 \%$ seguida del portugués con un 29,15 y el inglés con un $16,58 \%$, en CT predomina el inglés con el 69,71\%, seguido del Español con el $16,08 \%$ y del portugués que figura como tercera lengua con solo un 6,03\%. (tabla 2)

El predominio del español y del portugués en $\mathrm{CH}$ ocurre como consecuencia de la temática de las tesis. En CT las tesis tratan más de temas especializados y así utilizan más la literatura especializada publicada en los más diversos rincones del mundo.

Aunque con porcentajes muy bajos, el francés es la cuarta lengua más utilizada en las citas de las dos áreas seguida del italiano.

Con respecto a las otras lenguas en $\mathrm{CH}$ se ha constatado citas en checo, ruso, catalán y alemán mientras en las tesis de CT han citado más el Alemán y el Catalán.

El que se puede inferir que la lengua de la literatura citada depende del área y de la temática de la tesis y que el inglés es la lengua de mayor divulgación en el área técnica, mientras en el área de las humanidades hay una mayor dispersión en el uso de lenguas, principalmente de las lenguas latinas.
Las citas en español son en la mayoría de documentos publicados en España, principalmente en Madrid, seguidos de México, Argentina y otros países latinoamericano. Pero un buen porcentaje de estas son citas de documentos traducidos de otras lenguas al español, principalmente del inglés, el que reitera su condición de lengua oficial de la comunicación científica.

Las citas en inglés emanan de documentos publicados principalmente en los Estados Unidos, seguido del Reino Unido y de muchos otros países del mundo. Las citas en portugués proviene más de documentos publicados en Brasil, principalmente de la región sureste y, mayoritariamente, de São Paulo y Rio de Janeiro.

TABLA 2

Lengua de las citas

\begin{tabular}{lrrrr}
\hline Área/ & CH & $\%$ & CT & \% Lengua \\
\hline Ing. & 1284 & 16,58 & 4193 & 69,7 \\
Esp. & 3939 & 50,87 & 967 & 16,08 \\
Port. & 2257 & 29,15 & 363 & 6,03 \\
Fra. & 192 & 2,48 & 347 & 5,77 \\
Ita. & 35 & 0,45 & 97 & 1,6 \\
Otras & 37 & 0,47 & 48 & 0,80 \\
TOTAL & 7744 & 100 & 6015 & 100 \\
\hline
\end{tabular}

En cuanto a los tipo de documentos citados, en las tesis del área de $\mathrm{CH}$ predominan los libros, seguido de los artículos y en el área de CT predomina los artículos, seguido de los de trabajos de encuentros que incluye: Actas de Reuniones, trabajos de congresos, Seminarios, y trabajos presentados en todo tipo de acontecimientos, incluso Workshop.

En el área de $\mathrm{CH}$ el porcentaje de citas de Libros es de un $60,20 \%$, mientras que las citas a Artículos ocupan un $20,90 \%$ seguido de otros tipos de documentos con un $12,55 \%$, Informes con un 3\%, Encuentros con un 2,32\% y Tesis con sólo el 1,03\%. 
De todas las citas del área de CT el 55,44\% son de Artículos, mientras el porcentaje de Libros es de un 20,70\%, seguido de Encuentros con un 10,06\%, Informes con un $7,20 \%$, otros tipos con un $3,77 \%$ y Tesis con solo un $2,83 \%$. (Tabla 3 )

Las citas a Otros Tipos de material están muy diversificadas en función de la especialidad pero los tipos de documentos más citados en las dos áreas son los catálogos, datos estadísticos, diccionarios, documentos, entrevistas, proyectos normas y trabajos mecanogrfiados.

Se ha observado que las tesis son más citadas en la especialidad de Agrónomos y que la mayoría hacen referencia a tesis de sus colegas brasileños.

Se han encontrado diferencias significativa en las dos áreas y se puede inferir que el tipo de documento citado depende del área y de la temática de la tesis.

En cuanto a la cronología de las citas, el área de CT a pesar de las diferencias entre las especialidades, tiende a citar una literatura más actualizada que en $\mathrm{CH}$.

En el área de $\mathrm{CH}$ las citas de libros son las más antiguas, mientras que las correspondientes a artículos están más actualizadas. En cuanto a CT, las citas de informes y tesis son más actuales y al contrario de lo que cabría esperar, las citas de artículos son más antiguas.

En CH la especialidad más actual es Derecho, pero esto considerando que se han excluido las citas de siglos anteriores. Por otro lado, las que presentan mayor nivel de obsolescencia son las citas en Geografía y Historia, Psicología, Filosofía y Filología. En CT las citas mas actualizadas están en las carreras de Industriales y Telecomunicaciones, mientras las más antiguas están en Agrónomos.

Aunque las dos áreas tiendan a citar la literatura más actualizada y la edad más común sea de cinco años, los porcentajes de citas a documentos publicados con menos de 5 años no alcanzan el índice determinado por Price (cuarenta y tres por cien) que asegura la solidez de una ciencia.

Se puede concluir, que el nivel de actualización y obsolescencia es diferente no sólo de una área a otra, sino también entre especialidades, y depende tanto de la especialidad como del tipo de documento citado.
TABLA 3

Tipo de documentos citados

\begin{tabular}{lrrrr}
\hline ÁREA/ & CH & $\%$ & CT & $\%$ TIPO \\
\hline Artículos & 1619 & 20,90 & 3335 & 55,44 \\
Libros & 4662 & 60,20 & 1245 & 20,70 \\
Tesis & 80 & 1,03 & 170 & 2,83 \\
Informes & 231 & 3,00 & 433 & 7,20 \\
Encuentros & 180 & 2,32 & 605 & 10,06 \\
Otros & 972 & 12,55 & 277 & 3,77 \\
& & & & 100 \\
TOTAL & 7744 & 100 & 6015 & \\
\hline
\end{tabular}

Las tesis de CT tienden a citar más a los directores de tesis, que las de $\mathrm{CH}$. Pero las citas a los directores en CT no ocurren obligatoriamente en las tesis de los respectivos orientados, sino que están dispersas en varias tesis de la misma especialidad.

En el área de CT, los doctores tienden más a hacer uso de la autocita que en $\mathrm{CH}$. Mientras en $\mathrm{CH}$ las autocitas son más de libros, en CT, predominan la autocitas a trabajos de congresos. Estos, son en casi su totalidad, trabajos en colaboración con sus directores de tesis y presentados en congresos internacionales.

Aunque en bajos porcentajes, en las dos áreas se han constatado autocitas de tesis de Maestría y de trabajos internos mecanografiados o sin publicar. Queda saber si estos trabajos llegaron a ser publicados.

Aquí se hace patente que aunque las citas están dotadas de gran variedad, desigualdad, y fallos, así mismo éstas proporcionaron datos suficientes para concluir, que los patrones de citas de las tesis, a pesar de las diferencias entre especialidades, mantienen una cierta semejanza entre sí, dentro de cada una de las dos áreas.

De acuerdo con las variables analizadas y en resumen de las conclusiones, se puede afirmar que en el área de $\mathrm{CH}$ hay más discrepancias, mientras en CT los resultados son más uniformes.

Todavía no hay otros trabajos de investigación semejantes a este estudio, es decir, no se han encontrado estudios que versen sobre las tesis brasileñas en el extranjero, tampoco en España. Por eso no es posible hacer comparaciones. 
Aunque sea difícil determinar el «origen nacional» de un determinado saber científico, no es imposible documentar la producción científica de un determinado país. Documentar las tesis doctorales no sólo registra una parcela de la producción científica sino que también garantiza el registro de una producción de alta calidad, considerando que las tesis están sujetas a un sistema de evaluación formal.

Por el alto porcentaje de doctores que obtuvieron becas de instituciones brasileñas para cursar el doctorado y teniendo en cuenta el compromiso profesional que el becario brasileño asume para retornar a Brasil al concluir el doctorado, se puede afirmar que aunque se de una evasión de la producción científica brasileña, no ocurre lo mismo con los investigadores.

El resultado más importante de este estudio es tener documentada una parte de la producción científica brasileña en España, a través de las tesis doctorales que representan una parcela de la colaboración de los brasileños al conocimiento científico en general, y a la producción científica española.

Así se puede afirmar que la estancia de los brasileños en España, aún modestamente, ha contribuido a una mayor cooperación entre investigadores brasileños y españoles.

Esto se comprueba por los trabajos presentados, principalmente en congresos internacionales, por los brasileños en colaboración con sus directores de tesis, que reitera así los resultados de otros estudios, que analizan artículos de revistas y consideran a Brasil como el país de América Latina con mayor número de trabajos publicados en colaboración con otros países.

Se esperar que los acuerdos de cooperación firmado recientemente entre las universidades españolas y brasileñas vengan a incrementar el intercambio científico entre Europa y América Latina, y que estudios de esa naturaleza sean llevados a cabo en otros países y así se pueda documentar cada vez más la Producción científica brasileña en el extranjero.

Considerando el tratamiento sigilos dado a las tesis, en la mayoría de las universidades españolas, que mas se asemeja al tratamiento exigido para la preservación de documentos de inventos tecnológicos, es decir, de Patentes, se puede inferir que las tradicionales normativas y leyes españolas dificultan e impiden el proceso de divulgación del conocimiento y la transferencia de la información registrada en las tesis.
Así como expresión del interés por mejorar el proceso de investigación y el acceso a éste tipo de documento, se sugiere que se preserve y garantice el derecho del autor de las tesis divulgándolas debidamente. «El conocimiento para ser científico debe ser de conocimiento público». Por lo tanto las tesis deben estar disponibles para que sean consultadas y citadas.

Se espera, aunque modestamente, que este trabajo pueda contribuir teóricamente al desarrollo de los estudios en el campo de la Documentación y en términos prácticos lleve a las instituciones brasileñas hacer un diseño de la Producción Científica Brasileña en España además de ayudar a los futuros candidatos brasileños al doctorado a conocer, por lo menos en parte, la realidad del doctorado en universidades españolas.

\section{REFERÊNCIAS BIBLIOGRÁFICAS}

1. AMAT, Nuria. De la información al saber. Madrid: Promociones y Publicaciones universitarias. 1992, 135 p. BRASIL. Presidencia da República. SEPLAN. II Plano Nacional de Desenvolvimento, 1975. 79. Rio de Janeiro: IBGE, 1974.

2. CNPq registra 23 mil cientístas. Jornal da Ciência hoje. Rio de Janeiro, v. 9 , n. 303, p. 5, 1994.

3. JORNAL DA CIÊNCIA HOJE. Rio de Janeiro: SBPC, ano 9, n. $307,1994$.

4. FERREIRO ALÀEZ, Luis. Bibliometría: Análisis Bivariante. Madrid: Editora Eypasa, 1993, 480 p.

5. LANCASTER, F.W. \& CARVALHO, M.B.P. «Cientista brasileiro publica no exterior: en que países, en que revistas, sobre que assuntos». Ciencia e Cultura. v. 34, n. 35, 1982, pp. 672-634.

6. NASCIMENTO, Maria de Jesus. Estudo da produção científica brasileira na área nuclear no período 1970/1979. Rio de Janeiro: Univesidade Federal do Rio de Janeiro/Instituto Brasileño de Informação en Ciencia e Tecnologia. 1983, 209 p. (Diss. Mestrado)

7. PRICE, D. de Solla. Litle Science, Big Science. New York: Columbia Univ. Press. 1963, 117 p.

8. REDIAL. Red europea de Información y Documentación sobre América Latina. Repertorio de tesis Europeas sobre América latina 1980 - 1990. Madrid: Quinto Centenario, 1992, 429 p. SIERRA BRAVO, R. Tesis doctorales y trabajos de investigación científica. Tercera edición, revisada y ampliada. Madrid: Editorial Paraninfo. 1994, 497 p. 